\title{
Vertical Distribution of Suspended Sediment under Steady Flow: Existing Theories and Fractional Derivative Model
}

\author{
Shiqian Nie, ${ }^{1,2}$ HongGuang Sun, ${ }^{2}$ Yong Zhang, ${ }^{3}$ Dong Chen, ${ }^{4}$ Wen Chen, ${ }^{2}$ \\ Li Chen, ${ }^{5}$ and Sydney Schaefer ${ }^{3}$ \\ ${ }^{1}$ College of Water Conservancy and Hydropower Engineering, Hohai University, Nanjing, Jiangsu 210098, China \\ ${ }^{2}$ State Key Laboratory of Hydrology-Water Resources and Hydraulic Engineering, Institute of Soft Matter Mechanics, \\ College of Mechanics and Materials, Hohai University, Nanjing, Jiangsu 210098, China \\ ${ }^{3}$ Department of Geological Sciences, University of Alabama, Tuscaloosa, AL 35487, USA \\ ${ }^{4}$ Key Laboratory of Water Cycle and Related Land Surface Processes, Institute of Geographic Sciences and Natural Resources Research, \\ Chinese Academy of Sciences, Beijing 100101, China \\ ${ }^{5}$ Division of Hydrologic Sciences, Desert Research Institute, Las Vegas, NV 89119, USA
}

Correspondence should be addressed to HongGuang Sun; shg@hhu.edu.cn

Received 28 March 2017; Revised 10 May 2017; Accepted 24 May 2017; Published 28 June 2017

Academic Editor: Hengfei Ding

Copyright (C) 2017 Shiqian Nie et al. This is an open access article distributed under the Creative Commons Attribution License, which permits unrestricted use, distribution, and reproduction in any medium, provided the original work is properly cited.

\begin{abstract}
The fractional advection-diffusion equation (fADE) model is a new approach to describe the vertical distribution of suspended sediment concentration in steady turbulent flow. However, the advantages and parameter definition of the fADE model in describing the sediment suspension distribution are still unclear. To address this knowledge gap, this study first reviews seven models, including the fADE model, for the vertical distribution of suspended sediment concentration in steady turbulent flow. The fADE model, among others, describes both Fickian and non-Fickian diffusive characteristics of suspended sediment, while the other six models assume that the vertical diffusion of suspended sediment follows Fick's first law. Second, this study explores the sensitivity of the fractional index of the fADE model to the variation of particle sizes and sediment settling velocities, based on experimental data collected from the literatures. Finally, empirical formulas are developed to relate the fractional derivative order to particle size and sediment settling velocity. These formulas offer river engineers a substitutive way to estimate the fractional derivative order in the fADE model.
\end{abstract}

\section{Introduction}

The vertical distribution of suspended sediment concentrations in steady turbulent flow is an important measure when evaluating the suspended flux in natural rivers and canals [13]. The physical mechanism of a steady sediment suspension distribution is a dynamic equilibrium of vertical fluxes between downward sediment settling and upward turbulent diffusion. Nonequilibrium of the two opposing processes may result in erosion or deposition, which consequently brings environmental issues such as water and soil erosion and reservoirs deposition.

The theories of gravity, diffusion, mixing, energy dissipation, similarity, and stochastic models have been applied to study the vertical diffusion of sediment [4-7]. The theories of gravity and diffusion, among others, are the most frequently used. Yet, the gravity theory has been questioned due to the ambiguous definition of "suspension energy" [8]. The diffusion theory has been more widely applied in recent decades since it explains many observations in laboratory and field settings [9-11]. In addition, the two-phase flow system, which has been applied to investigate a variety of problems of interest in fluvial hydraulics, may be described in terms of either macroscopic or microscopic methods [12-18].

The diffusion theory is derived from the traditional advection-diffusion equation (ADE) model by assuming Fick's first law for sediment diffusion in turbulence:

$$
\frac{\partial S}{\partial t}=\omega \frac{\partial S}{\partial y}+\varepsilon_{s y} \frac{\partial^{2} S}{\partial y^{2}}
$$


where $S\left[\mathrm{ML}^{3}\right]$ is the sediment volumetric concentration, $y[\mathrm{~L}]$ is the vertical coordinate, $\omega[\mathrm{L} / \mathrm{T}]$ is the sediment settling velocity, and $\varepsilon_{s y}\left[\mathrm{~L}^{2} / \mathrm{T}\right]$ is the sediment turbulent diffusion coefficient along the $y$ direction. When downward sediment settling and upward turbulent diffusion reach the balanced state, it results in

$$
\omega S+\varepsilon_{s y} \frac{\partial S}{\partial y}=0
$$

Here $\varepsilon_{s y}=\beta \varepsilon_{m}$, and $\varepsilon_{m}$ is the fluid eddy viscosity and $\varepsilon_{m}=\kappa u_{*}(1-y / h) y$ based on the Karman-Prandtl logarithmic velocity profile. The analytical solution of (2) yields the wellknown Rouse vertical concentration profile [19]:

$$
s=\frac{S}{S_{a}}=\left[\frac{h / y-1}{h / a-1}\right]^{\omega / \beta \kappa u_{*}}
$$

where $s$ is the relative sediment concentration, $S_{a}$ is a reference concentration at a given height $a$ above the riverbed, $h$ [L] is the water depth, $a$ [L] is the reference height, $\kappa$ [dimensionless] is the Von Karman constant, $u_{*}[\mathrm{~L} / \mathrm{T}]$ is the shear velocity, and $\beta$ [dimensionless] is a proportionality coefficient related to diffusion of sediment particles.

As a milestone in the history of sediment transport, the Rouse formula (3) has been widely used for decades [4, 8, 28]. However, limitations of this theory are also obvious: the sediment concentration is calculated as zero at the water surface and infinity at the riverbed. Vanoni also stated, in his classical manual [29], that the Rouse formula can only represent the shape of the distribution not the actual values in a predictive sense. Therefore, many researchers have put forward modifications or improvements on the Rouse formula $[8,16,20-23]$. Besides the formula investigated in the next section, there are many valuable works on this topic. For example, Greimann et al. [24, 30] offered both numerical and analytical expressions for concentration profiles, based on two-phase flow analysis. Bombardelli and Jha [31] and Jha and Bombardelli [32-34] established a framework composed of the complete two-fluid model, a partial two-fluid model, and a standard sediment-transport model and further discussed different models in describing three datasets. Toorman [35] derived Eulerian equations for the vertical flux and momentum of suspended particles in dilute sediment-laden openchannel flow in equilibrium using the two-fluid approach.

Recently, the fractional advection-dispersion equation (fADE) model has been developed to describe anomalous diffusion of sediment [21,36-38]. As an extension of the traditional advection-dispersion equation, the fADE model can describe the anomalous diffusive characteristics of suspended sediment in turbulence, for example, nonlocal displacement or superdiffusion in certain circumstances such as turbulence bursting. However, the advantages and parameter determination method of the fADE model in describing suspended sediment transport are still unclear [36-43]. In this study, we investigate the Rouse formula and six improved models (including the fADE model) with explicit expressions. A comparison of these models in describing previously published experimental data has been presented for illustrative purposes. Furthermore, this study develops empirical formulas for accessing the fractional derivative order in terms of particle sizes or sediment settling velocities. The aim is to help river engineers in estimating the vertical distribution of suspended sediment concentration via the fADE model in real-world applications.

\section{Improved Models Based on the Rouse Formula}

2.1. Model 1 (M1). To obtain a sediment concentration distribution formula, which can be applied throughout the flow region along the depth in natural rivers, Zhang [20] developed a vertical distribution formula of suspended sediment by solving the diffusion equation accompanied by the velocity distribution formula in sediment-laden flow:

$$
s=\frac{S}{S_{a}}=\exp \left[5.33 \frac{\omega}{\kappa u_{*}}\left(\arctan \sqrt{\frac{h}{y}-1}-1.345\right)\right] .
$$

2.2. fADE Model. Numerous studies have shown that particle dynamics in turbulent flow exhibits anomalous diffusive characteristics and can be well described using the fractional Fick's law [44]. Chen et al. [21] pointed out that turbulence bursting sometimes plays a key role in sediment diffusion and further proposed a fADE model using the fractional derivative to characterize nonlocal particle movement in steady turbulence:

$$
\omega S+\bar{\varepsilon}_{s y} \frac{\partial^{\alpha} S}{\partial y^{\alpha}}=0
$$

where $\alpha$ is the fractional derivative order $(0<\alpha \leq 1)$. Considering that the anomalous diffusion occurs in an entire water body, they replaced the depth-dependent coefficient $\varepsilon_{s y}$ with a depth-averaged diffusivity $\varepsilon_{s y}$ in (5). New $\bar{\varepsilon}_{s y}$ is obtained by integrating Rouse's [29] expression of $\varepsilon_{s y}$ from the reference height $a(a=0.05 h)$ to the water surface $(y=h)$ :

$$
\bar{\varepsilon}_{s y}=\frac{\int_{0.05 h}^{h} \kappa u_{*}(1-y / h) y d y}{0.95 h}=\frac{209 \kappa u_{*} h}{1200} .
$$

In model (5), the definition of fractional derivative is expressed as follows [45]:

$$
\frac{\partial^{\alpha} S}{\partial y^{\alpha}}=\frac{1}{\Gamma(1-\alpha)} \int_{a}^{t}(t-\tau)^{-\alpha} S^{\prime}(\tau) d \tau, \quad 0<\alpha \leq 1 .
$$

By employing the theoretical technique of fractional diffusion equation and property of Mittag-Leffler function, the analytical solution of (5) for sediment suspension can be written as follows [39]:

$$
s=\frac{S}{S_{a}}=E_{\alpha}\left[-\frac{\omega}{\varepsilon_{s y}}\left(y-y_{a}\right)^{\alpha}\right],
$$

in which the Mittag-Leffler function $E_{\alpha}(*)$ is expressed as

$$
E_{\alpha}(z)=\sum_{k=0}^{\infty} \frac{z^{k}}{\Gamma(\alpha k+1)} .
$$


2.3. Model 2 (M2). The Van Rijn formula is a frequently used method to describe the vertical distribution of suspended sediment. It is a piecewise distribution function for vertical sediment concentration, which considers the interaction between flow and sediment [46]. Zheng et al. [22] further developed a continuous sediment concentration distribution formula by modifying the Van Rijn formula. Then, the vertical distribution formula of suspended sediment can be written as

$$
s=\frac{S}{S_{a}}=\left\{\left(\frac{h-a}{a}\right) \frac{y}{h}\left[1+e^{4(y / h-1 / 2)}\right]\right\}^{-Z},
$$

where the suspension index $Z=\omega / \kappa u_{*}$.

2.4. Power Law Model. This model was introduced from the turbulent energy theory approach, which yields a sediment distribution expressed as follows [23]:

$$
s=\frac{S}{S_{a}}=\left[\frac{(1-y / h)(d u / d y)}{(1-a / h)(d u / d y)_{a}}\right]^{Z} .
$$

If we employ Prandtl's logarithmic velocity profile $(d u / d y=$ $\left.u_{*} / \kappa y\right)$ in formula (11), then the Rouse formula (3) can be achieved. But if we employ the power law type velocity profile in steady flow,

$$
\frac{u}{u_{\max }}=\left(\frac{y}{h}\right)^{n}
$$

where $u_{\max }$ is the maximum velocity and the exponent $n$ relates to the Reynolds number and the drag coefficient. In a general case, $n$ equals $1 / 6 \sim 1 / 10$, such as $n=0.125$ [6]. We then obtain a new expression of the vertical sediment distribution by substituting formula (12) into (11):

$$
s=\frac{S}{S_{a}}=\left[\left(\frac{h-y}{h-a}\right)\left(\frac{a}{y}\right)^{1-n}\right]^{Z},
$$

where the exponent $Z=\omega / \kappa u_{*}$.

2.5. Wang Model. A typical theoretical model for the particle concentration distribution is given by Wang and coworkers using the kinetic theory for two-phase flow [14]:

$$
s=\frac{S}{S_{a}}=\left(\frac{y}{a}\right)^{\zeta-1} e^{-Z[(y-a) / h]} .
$$

If $Z=6\left(\omega / \kappa u_{*}\right)$ and $\zeta=1$, the above formula is converted into

$$
s=\frac{S}{S_{a}}=e^{-6\left(\omega / \kappa u_{*}\right)[(y-a) / h]},
$$

which is named as the Lane-Kalinske formula.

2.6. Two-Phase Flow Model. Although some two-phase flow models describe the dynamics of suspended sediment well, they have no explicit expression [32, 33]. For small particles and dilute concentrations in sediment-laden flow (i.e.,
$S t_{b} / \kappa \eta \ll 1$ and $S \ll 0.1$ ), we can use a simplified model of two-phase flow [24, 30]:

$$
s=\frac{S}{S_{a}}=\left[\frac{\eta-1}{\eta} \cdot \frac{a}{a-1}\right]^{Z / \gamma_{c}},
$$

where $\eta=y / h, a$ is the reference height, $Z=\omega / \kappa u_{*}$, and $\gamma_{c}$ is the coefficient accounting for the crossing-trajectory and continuity effects.

\section{Comparison and Discussion}

A summary of seven existing models including the Rouse formula and its extensions is shown in Table 1. Based on the observation of Table 1, it is clear that the six extended models improve the applicability of the Rouse formula and overcome the main description drawbacks at the riverbed and surface (except for the power law model and the twophase flow model at the surface). As shown in Table 1, M1, M2, and Wang model have less parameters, while the fADE model, the power law model, and two-phase flow model contain one more parameter than the other models. M2 is obtained by modifying the Van Rijn model, among others. In the Wang model, a particle velocity distribution function is obtained in the equilibrium state or in a dilute steady state for a particle in two-phase flow, and then a theoretical model for the particle concentration distribution is derived from the kinetic theory. The power law model and M1 are obtained by a combination of the velocity distribution formula. In the two-phase flow model, a theoretical expression is obtained by assuming dilute concentrations and small particles. The fADE model is achieved by employing a fractional derivative to characterize anomalous diffusion in turbulence.

To further explore the feature of the above formulas in characterizing the vertical distribution of sediment suspension, here we investigate the vertical concentration profiles obtained using the above models. Figure 1 shows significant differences in the shape of the vertical profile for the different models. Generally speaking, the concentration calculated by the fADE model and Wang model under $0.05 \mathrm{~h}$ changes slowly, but the other models change dramatically, especially for the Rouse model. The M2, power law model, and Rouse model are coincident under $0.05 h$. The M1, the power law model, and the two-phase flow model provide similar results as the Rouse formula near the water surface, in which the concentration tends to be zero. The Rouse model, M1, the fADE model, Wang model, M2, the power law model, and the two-phase flow model offer a similar description of the vertical distribution between heights $0.05 h$ and $0.9 h$.

When the settling velocity $\omega$ increases from 0.01 to 0.04 (Figure 2), the concentration profiles of different models exhibit more nonuniform distribution features. The water surface concentration calculated by the fADE model is larger than the other models, while the concentration using the Rouse model, M1, Wang model, M2, the power law model, and the two-phase flow model tends to be zero. It should also be noted that all of the calculated concentration profiles of the existing models have a similar decay trend under the height of $0.05 h$ and the corresponding variation tendency 


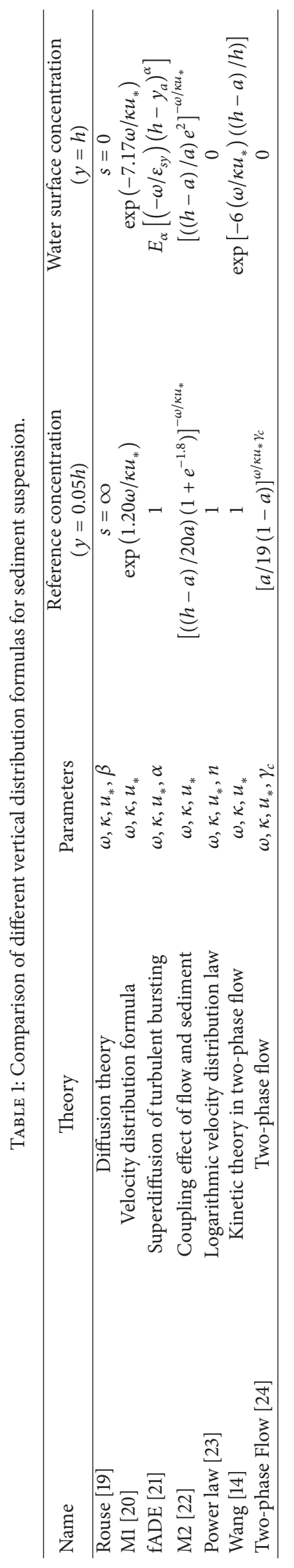




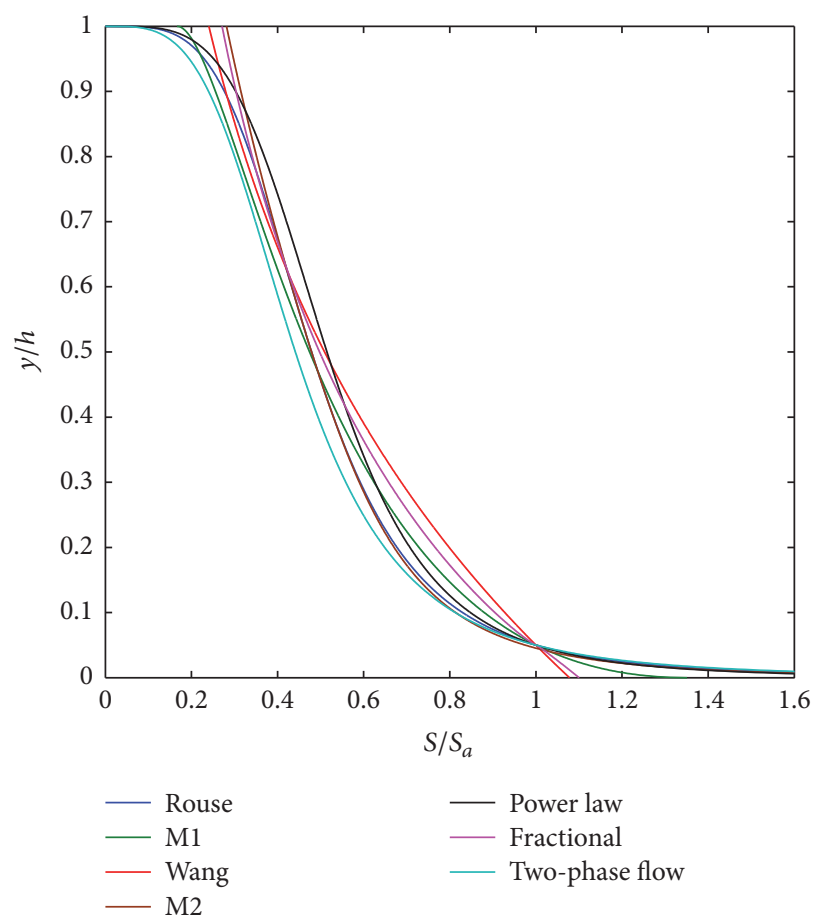

FIGURE 1: Numerical results of different models in describing the vertical distribution of sediment suspension. Von Karman constant $\kappa=0.4$, shear velocity $u_{*}=0.10$, sediment settling velocity $\omega=0.01$, water depth $h=1.0, a=0.05 h$, and $\alpha=0.9$ in the fADE model. For description simplicity, units are not used for parameters in this figure.

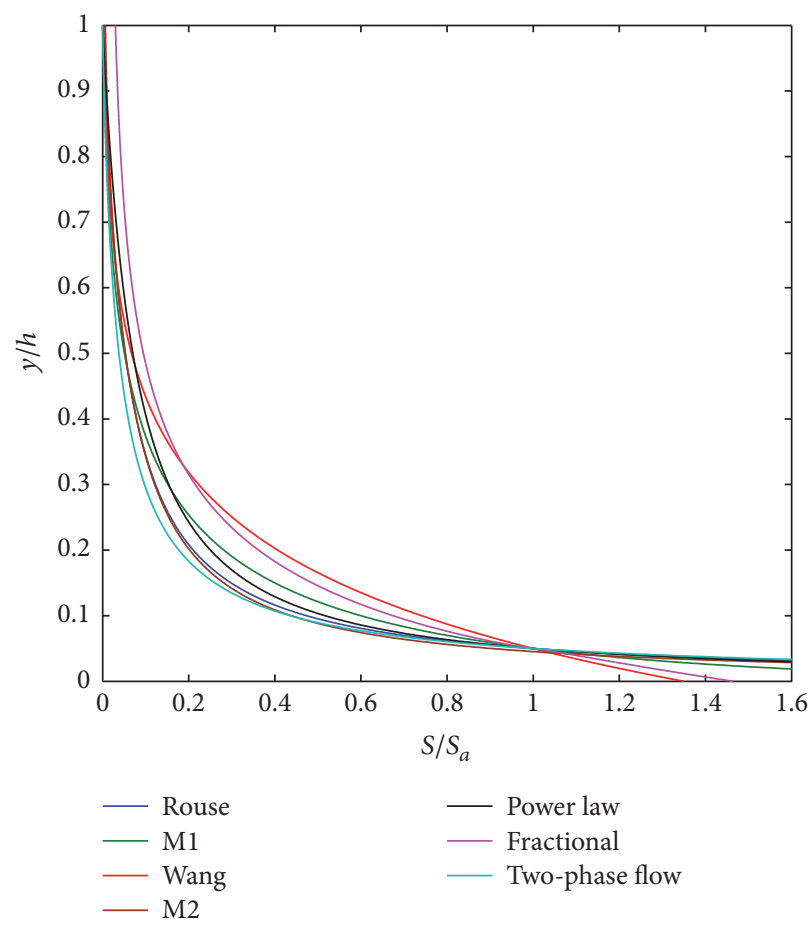

FIGURE 2: Numerical results of different models in describing the vertical distribution of sediment suspension. Von Karman constant $\kappa=0.4$, shear velocity $u_{*}=0.10$, sediment settling velocity $\omega=0.04$, water depth $h=1.0, a=0.05 h$, and $\alpha=0.9$ in the fADE model. For description simplicity, units are not used for parameters in this figure. 


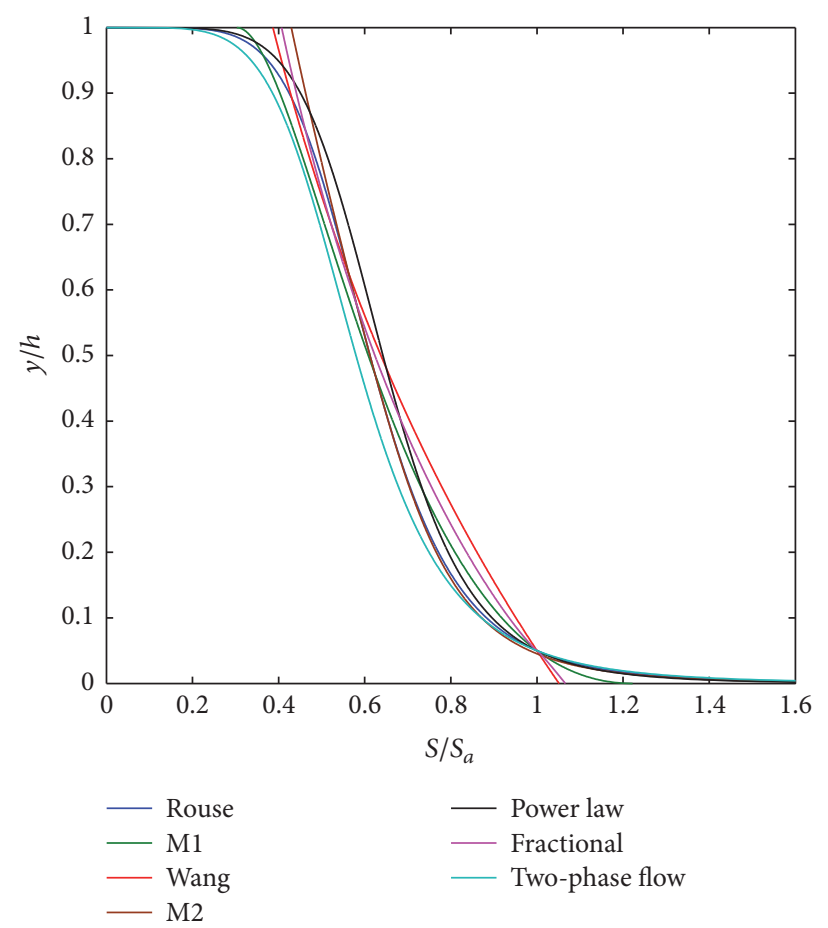

FIGURE 3: Numerical results of different models in describing the vertical distribution of sediment suspension. Von Karman constant $\kappa=0.4$, shear velocity $u_{*}=0.15$, sediment settling velocity $\omega=0.01$, water depth $h=1.0, a=0.05 h$, and $\alpha=0.9$ in the fADE model. For description simplicity, units are not used for parameters in this figure.

keeps consistent with an increasing settling velocity. The comparison results (Figures 1-3) also show that shear velocity is not a critical parameter in controlling the decay pattern of the concentration profiles. Hence, it can be concluded that shear velocity plays a noncritical role in determining the vertical concentration distribution when compared with the other factors.

To test the efficiency of the above models in describing the vertical distribution of suspended sediment, here we use two groups of experimental data from Einstein and Chien [27] and Lyn [26]. The experiments were carried out with natural sands in two-dimensional, fully developed, and steady open-channel flows. In the experiments, coarse sand (with a diameter of $d=1.3 \mathrm{~mm}$ ) was used for runs $\mathrm{S}-2$ and S-3, and medium sand $(d=0.94 \mathrm{~mm})$ for runs S-8 and S9 in Einstein and Chien [27], while 1965EQ $(d=0.19 \mathrm{~mm})$ and 2565EQ $(d=0.24 \mathrm{~mm})$ were in Lyn [26]. Details of the experiments can be found in the related references $[17,18,47]$. The comparison results of different vertical distribution formulas in fitting the experimental data are drawn in Figures 4-6. As shown in Figures 4 and 5, it is clear that most extensions better describe the vertical distribution of sediment suspension than the Rouse formula. Figures 4 and 5 show the concentration distributions of runs S-2 and S3 for coarse sand $(d=1.30 \mathrm{~mm})$ and S-8 and S-9 for medium sand $(d=0.94 \mathrm{~mm})$, respectively. The fractional derivative order $\alpha$ is numerically calculated using the experimental data and was eventually determined as 0.98 for S-2, 0.94 for S-3, and 0.88 for S- 8 and S-9, respectively. Generally speaking, the fADE model and the Wang model are better than the other models in fitting the experimental data. Moreover, the fADE model offers a better description of the vertical distribution near the riverbed $(0.05 h<y<0.10 h)$. Similar results are also found in Figure 5 for medium sand $(d=0.94 \mathrm{~mm})$.

Figure 6 indicates that the fADE model is the best one in the seven models to fit the experimental data with natural sand conducted by Lyn [26]. Moreover, the fADE model and Wang model offer a better description of the vertical distribution near the riverbed $(0.05 h<y<0.10 h)$ than the other models.

It is noteworthy that the Rouse formula with parameter $\beta \neq 1$ has also been used to describe the concentration profile. Figure 7 provides the experimental data fitting result of the Rouse formula with $\beta \neq 1$. Clearly, it gives a better agreement with experimental data than the basic Rouse formula, especially at the bottom region. However, since it is necessary to adjust the parameter $\beta$ frequently to coordinate the suspended sediment concentration profiles with the measured data, this formula is not easy to use in realworld applications.

\section{Fractional Index of the fADE Model and Hydraulic Parameters}

The fractional index is a key parameter in the fADE model to characterize the impact of turbulent bursting on sediment suspension. Therefore, the determination of the fractional index is a critical issue in real-world applications of the fADE model. Here we investigate the relationship between 


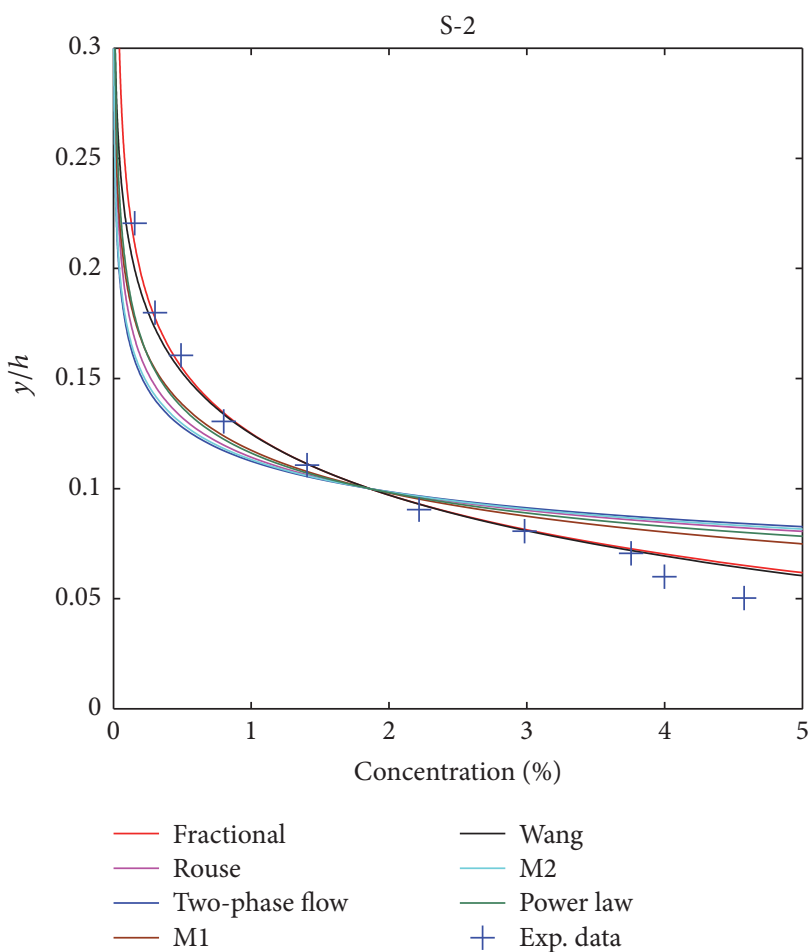

(a)

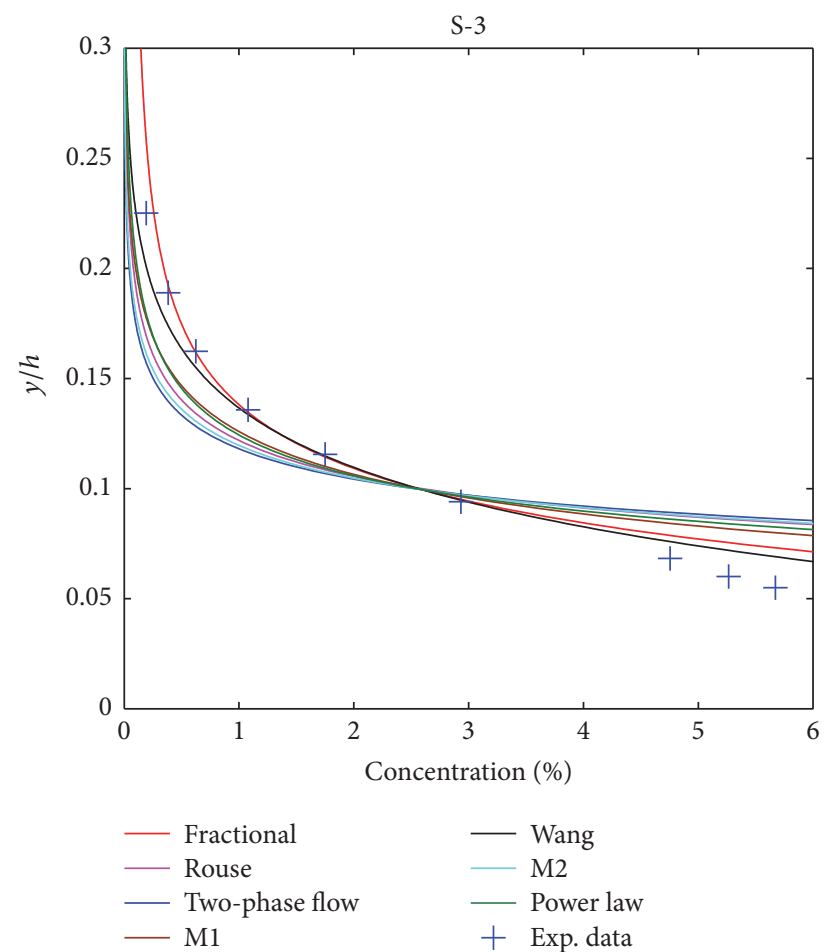

(b)

Figure 4: Results of the different models in describing the experimental data of Einstein and Chien [27]. (a) S-2 ( $d=1.3 \mathrm{~mm})$, the fractional derivative order $\alpha=0.98$, and the reference height $a=0.1 \mathrm{~h}$. (b) S-3 $(d=1.3 \mathrm{~mm})$, the fractional derivative order $\alpha=0.94$, and the reference height $a=0.1 h$.

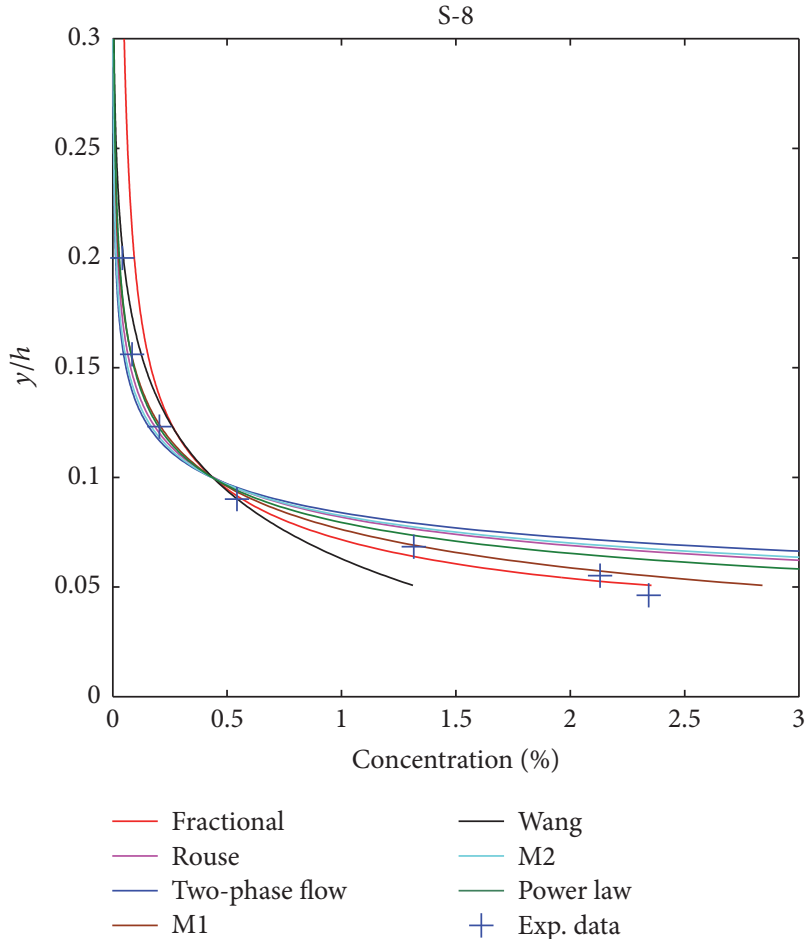

(a)

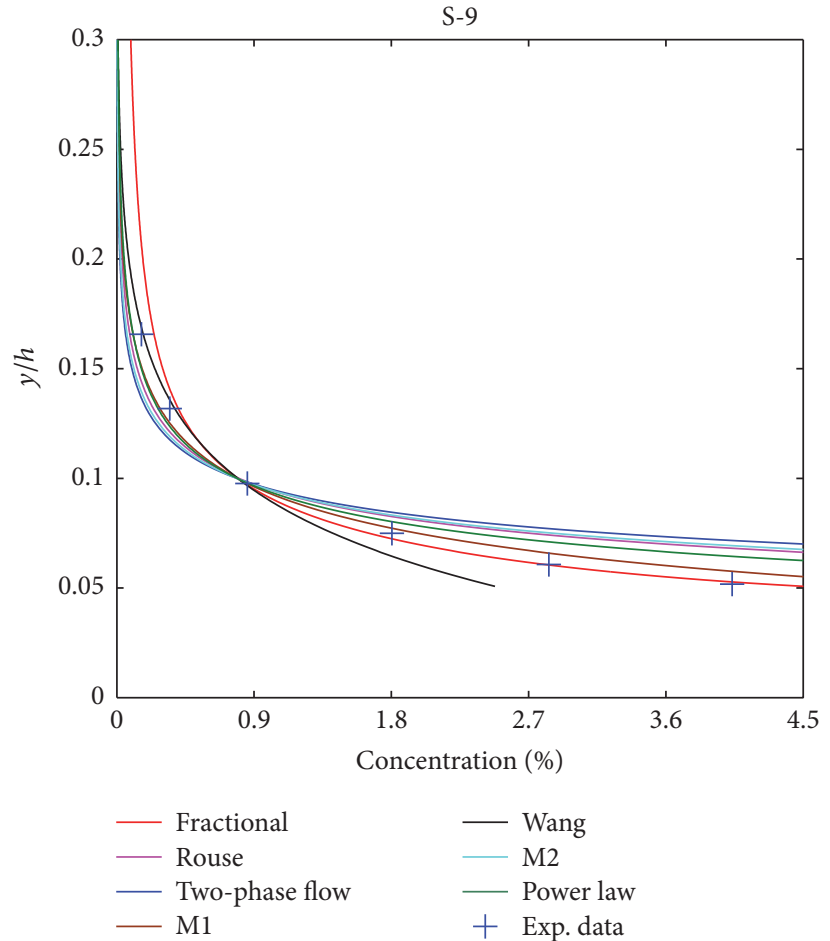

(b)

Figure 5: Results of the different models in describing the experimental data of Einstein and Chien [27]. (a) S-8 ( $d=0.94 \mathrm{~mm})$, the fractional derivative order $\alpha=0.88$, and the reference height $a=0.1 h$. (b) S-9 $(d=0.94 \mathrm{~mm})$, the fractional derivative order $\alpha=0.88$, and the reference height $a=0.1 h$. 


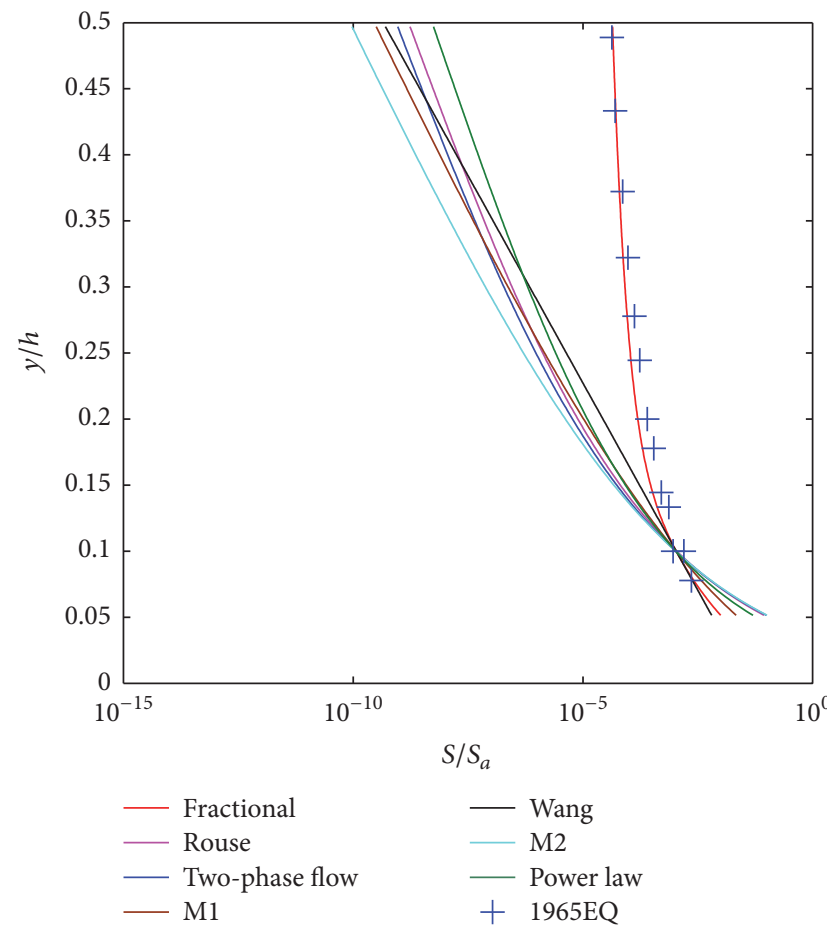

(a)

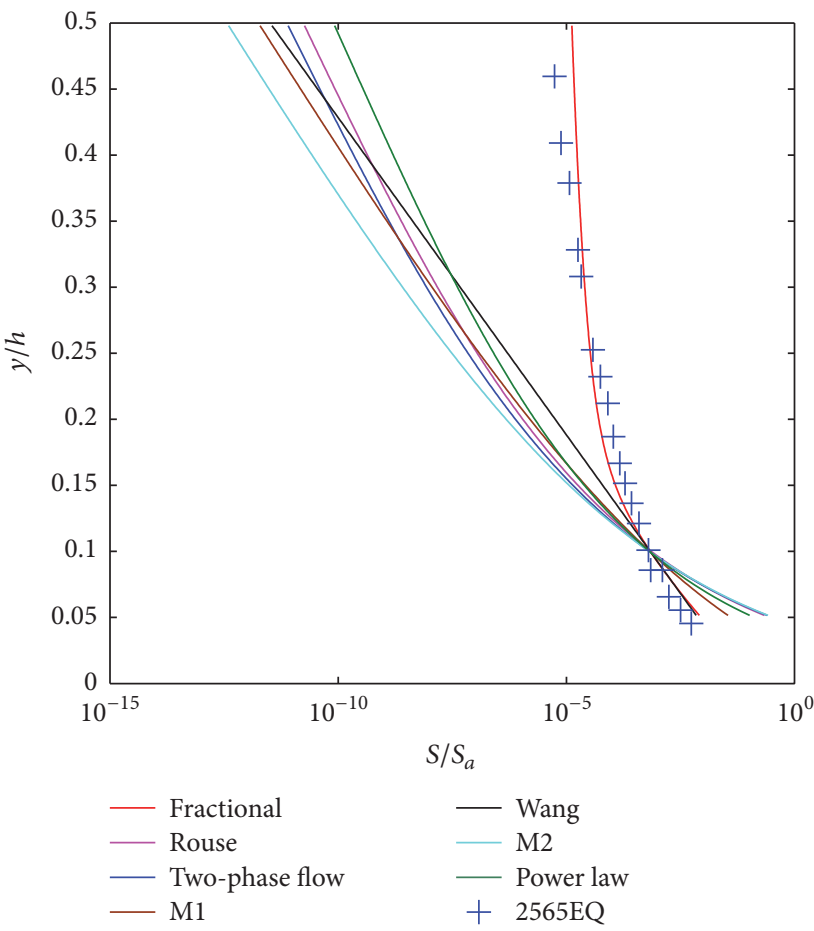

(b)

FIGURE 6: Results of the different models in describing the experimental data of Lyn [26]. (a) 1965EQ ( $d=0.19$ mm), the fractional derivative order $\alpha=0.93$, and the reference height $a=0.1 h$. (b) 2565EQ $(d=0.24 \mathrm{~mm})$, the fractional derivative order $\alpha=0.97$, and the reference height $a=0.1 h$.

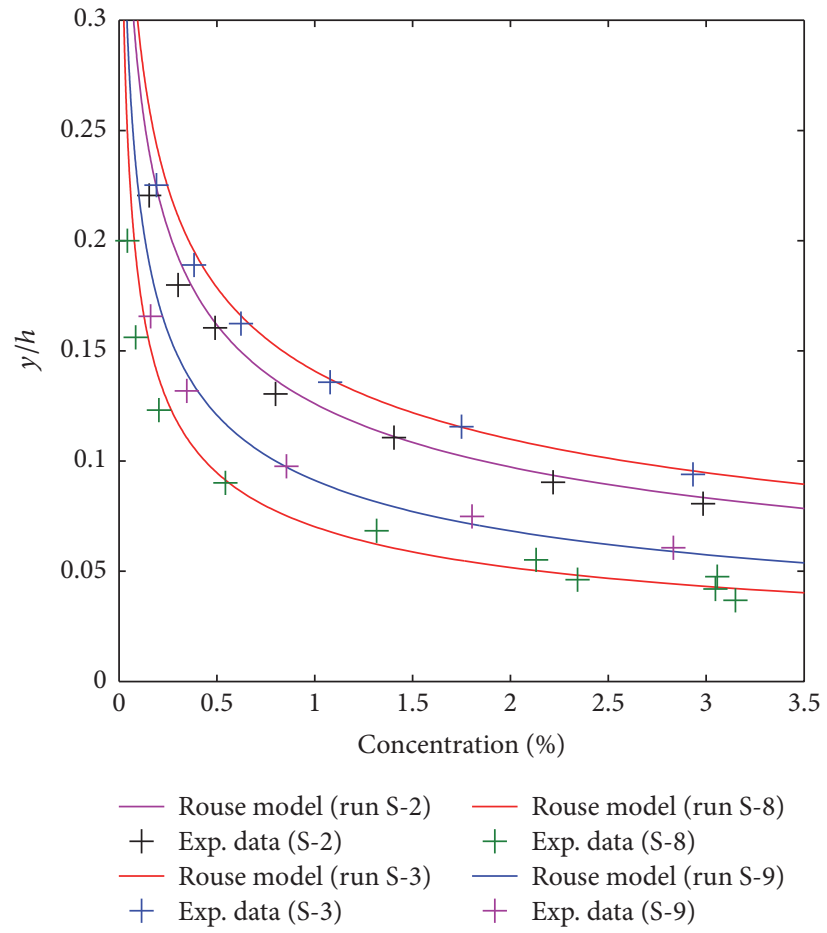

Figure 7: Results of the Rouse model in describing the experimental data of Einstein and Chien [27] with $\beta=1.75, \mathrm{~S}-2, \mathrm{~S}-3$ ( $d=1.3 \mathrm{~mm}$ ), and S-8, S-9 $(d=0.94 \mathrm{~mm})$. 
TABLE 2: The best fit fractional index $\alpha$ in the fADE model and corresponding hydraulic parameters, $d$-the particle size, and $\omega$-the settling velocity. Here, “A” denotes Chen et al. [21], "B" denotes Coleman [25], "C" denotes Lyn [26], and "D" denotes Einstein and Chien [27].

\begin{tabular}{lccc}
\hline Run number & Fractional index $\alpha$ & Particle size $d(\mathrm{~mm})$ & Settling velocity $\omega(\mathrm{m} / \mathrm{s})$ \\
\hline A1 & 0.095 & 0.035 & 0.000587 \\
A2 & 0.12 & 0.075 & 0.002681 \\
A3 & 0.20 & 0.175 & 0.013665 \\
A4 & 0.41 & 0.375 & 0.044323 \\
B1 & 0.55 & 0.105 & 0.00269 \\
B2 & 0.90 & 0.210 & 0.0101 \\
B3 & 0.75 & 0.420 & 0.03 \\
C1 & 0.86 & 0.150 & 0.016 \\
C2 & 0.93 & 0.190 & 0.023 \\
C3 & 0.97 & 0.240 & 0.031 \\
D1 & 0.80 & 0.274 & 0.035173 \\
D2 & 0.88 & 0.940 & 0.114689 \\
D3 & 0.94 & 1.300 & 0.140989 \\
\hline
\end{tabular}

the fractional index in the fADE model and hydraulic parameters. The experimental data used in this section comes from Chen et al. [21], Einstein and Chien [27], Lyn [26], and Coleman [25].

Model parameters listed in Table 2 indicate that the fractional index $\alpha$ increases with particle size $d$ and settling velocity $\omega$. Small fractional indexes for fine particles mean that superdiffusion behavior dominates sediment particle movement, since the finer particles have more chances to jump long distances during a turbulent bursting event. Meanwhile, the coarse particles are not as easily influenced by the turbulent bursting event and hence tend to exhibit normal diffusion behavior, resulting in the fractional index $\alpha$ close to 1 . Meanwhile, Table 2 also shows that the fractional index $\alpha$ is more sensitive to coarse or medium particles than fine particles. The best linear fitting functions, which can be used as empirical formulas, are offered in Figure 8. The figure depicts a linear relationship between the fractional index $\alpha$ and particle size $d$ for a given experimental condition. However, here we should point out that the fitting functions for different experiments are different, due to variations in the flow fields and the geometric structure of river sites.

The relationship between the fractional index $\alpha$ and settling velocity $\omega$ is similar to that of particle size. A particle's settling velocity is determined by the sediment gravity and diffusion, and it can partially represent the turbulence bursting effect on sediment movement. Figure 9 shows that a higher settling velocity corresponds to a larger fractional index $\alpha$, implying that the sediment diffusion behavior changes from superdiffusion to normal diffusion with an increase in settling velocity $\omega$. In addition, it is clear that the particle size and settling velocity have similar influences on the fractional index. The reason may lie in the close relationship between settling velocity and particle size, where a large particle size usually yields a high settling velocity. Meanwhile the fractional index $\alpha$ exhibits a better linear relationship with the settling velocity from data fitting results.

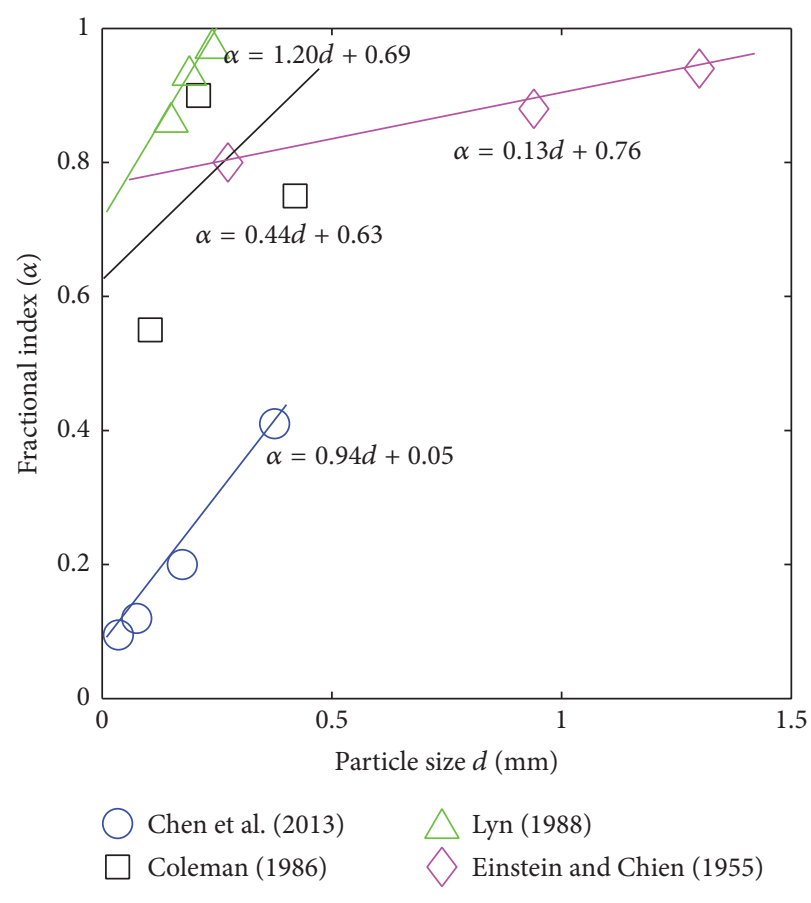

FIGURE 8: The relationship between the fractional index $\alpha$ in the fADE model and the particle size $d$. The best-fit functions are obtained using the least square fitting.

\section{Conclusions}

This study evaluates seven existing formulas, including the one deduced by the fADE model, in describing the vertical distribution of sediment suspension under the steadystate flow condition. Numerical simulation results indicate that the fADE model provides a good agreement with the experimental data of Einstein and Chien [27] and Lyn [26], compared with the other models. Moreover, the fractional index in the fADE model can be estimated using a linear 


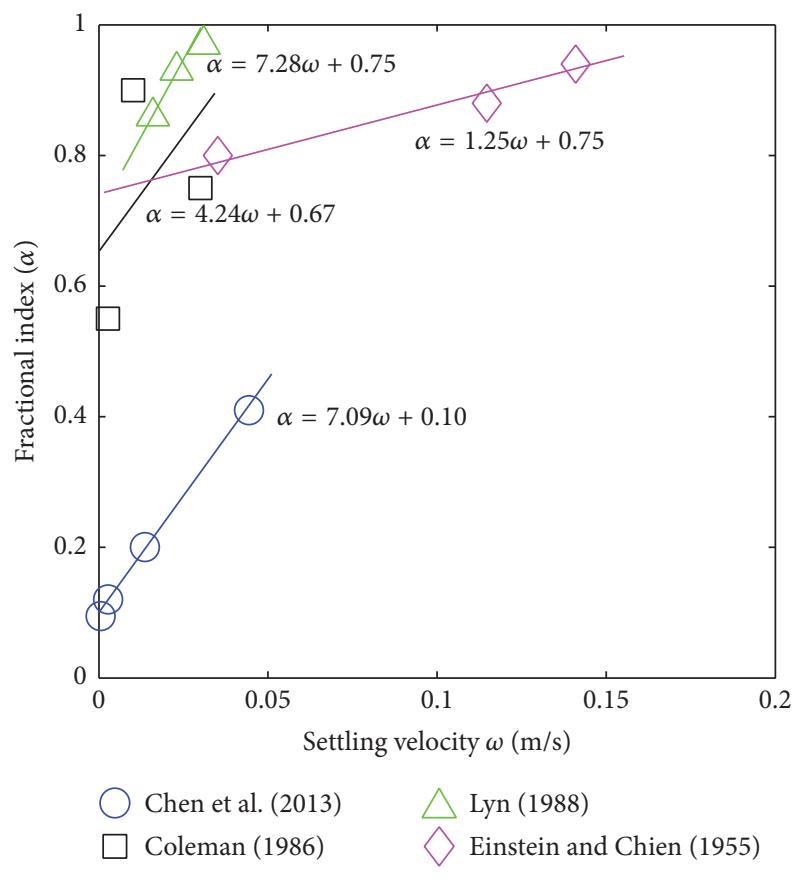

FIGURE 9: The relationship between the fractional index $\alpha$ in the fADE model and the settling velocity $\omega$. The best-fit functions are obtained using the least square fitting.

function of the particle size or settling velocity, based on existing experimental data reported in the literatures.

\section{Disclosure}

This work does not necessary reflect the view of the funding agency.

\section{Conflicts of Interest}

The authors declare that there are no conflicts of interest regarding the publication of this paper.

\section{Acknowledgments}

This work is supported by the National Natural Science Foundation of China under Grants nos. 11572112, 41628202, and 11528205.

\section{References}

[1] M. Cellino and U. Lemmin, "Influence of coherent flow structures on the dynamics of suspended sediment transport in open-channel flow," Journal of Hydraulic Engineering, vol. 130, no. 11, pp. 1077-1088, 2004.

[2] H. Depeweg and V. Néstor Méndez, "Sediment transport applications in irrigation canals," Irrigation and Drainage, vol. 51, no. 2, pp. 167-179, 2002.

[3] J. M. Turowski, D. Rickenmann, and S. J. Dadson, "The partitioning of the total sediment load of a river into suspended load and bedload: A review of empirical data," Sedimentology, vol. 57, no. 4, pp. 1126-1146, 2010.

[4] N. Chien and Z. H. Wan, Mechanics of sediment transport, Science Press, Beijing, China, 2003.

[5] Q.-C. Guo and Y.-C. Jin, "Modeling sediment transport using depth-averaged and moment equations," Journal of Hydraulic Engineering, vol. 125, no. 12, pp. 1262-1269, 1999.

[6] C. V. Alonso, "Stochastic models of suspended-sediment dispersion.," Journal of the Hydraulics Division, ASCE, vol. 107, no. 16307, pp. 733-757, 1981.

[7] J. R. Ni and G. Q. Wang, "Vertical sediment distribution," Journal of Hydraulic Engineering, vol. 117, no. 9, pp. 1184-1194, 1991.

[8] R. J. Zhang, J. H. Xie, and W. B. Chen, River Dynamics, Wuhan University Press, Wuhan, 2007.

[9] B. Ontowirjo and A. Mano, "A turbulent and suspended sediment transport model for plunging breakers," Coastal Engineering Journal, vol. 50, no. 3, pp. 349-367, 2008.

[10] J. Chauchat and S. Guillou, "On turbulence closures for twophase sediment-laden flow models," Journal of Geophysical Research: Oceans, vol. 113, no. 11, Article ID C11017, 2008.

[11] Q.-Q. Liu, A.-P. Shu, and V. P. Singh, "Analysis of the vertical profile of concentration in sediment-laden flows," Journal of Engineering Mechanics, vol. 133, no. 6, pp. 601-607, 2007.

[12] Z. Cao, L. Wei, and J. Xie, "Sediment-laden flow in open channels from two-phase flow viewpoint," Journal of Hydraulic Engineering, vol. 121, no. 10, pp. 725-735, 1995.

[13] G. Wang and X. Fu, "Mechanisms of particle vertical diffusion in sediment-laden flows," Chinese Science Bulletin, vol. 49, no. 10, pp. 1086-1090, 2004.

[14] G. Wang and J. Ni, "Kinetic theory for particle concentration distribution in two-phase flow," Journal of Engineering Mechanics, vol. 116, no. 12, pp. 2738-2748, 1990.

[15] G. Q. Wang and J. R. Ni, "The kinetic theory for dilute solid/liquid two-phase flow," International Journal of Multiphase Flow, vol. 17, no. 2, pp. 273-281, 1991.

[16] J. R. Ni, G. Q. Wang, and A. G. L. Borthwick, "Kinetic theory for particles in dilute and dense solid-liquid flows," Journal of Hydraulic Engineering, vol. 126, no. 12, pp. 893-903, 2000.

[17] D. Zhong, G. Wang, and Q. Sun, “Transport equation for suspended sediment based on two-fluid model of solid/liquid two-phase flows," Journal of Hydraulic Engineering, vol. 137, no. 5, pp. 530-542, 2011.

[18] X. Fu, G. Wang, and X. Shao, "Vertical dispersion of fine and coarse sediments in turbulent open-channel flows," Journal of Hydraulic Engineering, vol. 131, no. 10, pp. 877-888, 2005.

[19] H. Rouse, "Modern conceptions of the mechanics of fluid turbulence," Transactions of the American Society of Civil Engineers, vol. 102, no. 1, pp. 463-505, 1937.

[20] H. W. Zhang, "Distribution of suspended sediment concentration along depth in sediment laden flow," Journal of Sediment Research, no. 1, pp. 35-41, 1997, in Chinese.

[21] D. Chen, H. Sun, and Y. Zhang, "Fractional dispersion equation for sediment suspension," Journal of Hydrology, vol. 491, no. 1, pp. 13-22, 2013.

[22] J. Zheng, R. Li, and Q. Feng, "Vertical distribution of nearshore sediment concentration," Applied Mechanics and Materials, vol. 170-173, pp. 2272-2275, 2012.

[23] J. Z. Yuan and Y. J. Hui, "Concentration distribution formula derived by an exponential velocity distribution," Journal of Sediment Research, no. 4, pp. 99-102, 1992, in Chinense. 
[24] B. P. Greimann and F. M. Holly Jr., "Two-phase flow analysis of concentration profiles," Journal of Hydraulic Engineering, vol. 127, no. 9, pp. 753-762, 2001.

[25] N. L. Coleman, "Effects of Suspended Sediment on the Open-Channel Velocity Distribution," Water Resources Research, vol. 22, no. 10, pp. 1377-1384, 1986.

[26] D. A. Lyn, "A similarity approach to turbulent sediment-laden flows in open channels," Journal of Fluid Mechanics, vol. 193, pp. $1-26,1988$.

[27] H. A. Einstein and N. Chien, "Effects of heavy sediment concentration near the bed on velocity and sediment distribution," M. R. D. Sediment Series, Rep. No. 8, Univ. of California, Berkeley, Calif. and US Army Corps of Engineers, 1955.

[28] B. S. Mazumder and K. Ghoshal, "Velocity and concentration profiles in uniform sediment-laden flow," Applied Mathematical Modelling, vol. 30, no. 2, pp. 164-176, 2006.

[29] V. A. Vanoni, Sedimentation Engineering, American Society of Civil Engineers, 2006.

[30] B. P. Greimann, M. Muste, and F. M. Holly Jr., "Two-phase formulation of suspended sediment transport," Journal of Hydraulic Research, vol. 37, no. 4, pp. 479-499, 1999.

[31] F. A. Bombardelli and S. K. Jha, "Hierarchical modeling of the dilute transport of suspended sediment in open channels," Environmental Fluid Mechanics, vol. 9, no. 2, pp. 207-235, 2009.

[32] S. K. Jha and F. A. Bombardelli, "Two-phase modeling of turbulence in dilute sediment-laden, open-channel flows," Environmental Fluid Mechanics, vol. 9, no. 2, pp. 237-266, 2009.

[33] S. K. Jha and F. A. Bombardelli, "Toward two-phase flow modeling of nondilute sediment transport in open channels," Journal of Geophysical Research: Earth Surface, vol. 115, no. 3, Article ID F03015, 2010.

[34] S. K. Jha and F. A. Bombardelli, "Theoretical/numerical model for the transport of non-uniform suspended sediment in open channels," Advances in Water Resources, vol. 34, no. 5, pp. 577591, 2011.

[35] E. A. Toorman, "Vertical mixing in the fully developed turbulent layer of sediment-laden open-channel flow," Journal of Hydraulic Engineering, vol. 134, no. 9, pp. 1225-1235, 2008.

[36] D. N. Bradley, G. E. Tucker, and D. A. Benson, "Fractional dispersion in a sand bed river," Journal of Geophysical Research, vol. 115, no. F1, pp. 1-20, 2010.

[37] Y. Zhang, M. M. Meerschaert, and A. I. Packman, "Linking fluvial bed sediment transport across scales," Geophysical Research Letters, vol. 39, no. 20, Article ID L20404, 2012.

[38] H. G. Sun, D. Chen, Y. Zhang, and L. Chen, "Understanding partial bed-load transport: experiments and stochastic model analysis," Journal of Hydrology, vol. 521, pp. 196-204, 2015.

[39] I. Podlubny, Fractional Differential Equation, Academic Press, New York, USA, 1999.

[40] M. M. Meerschaert and C. Tadjeran, "Finite difference approximations for fractional advection-dispersion flow equations," Journal of Computational and Applied Mathematics, vol. 172, no. 1, pp. 65-77, 2004.

[41] C. Li and W. Deng, "Remarks on fractional derivatives," Applied Mathematics and Computation, vol. 187, no. 2, pp. 777-784, 2007.

[42] F. Liu, V. Anh, and I. Turner, "Numerical solution of the space fractional Fokker-Planck equation," vol. 166, no. 1, pp. 209-219.

[43] R. Schumer, D. A. Benson, M. M. Meerschaert, and S. W. Wheatcraft, "Eulerian derivation of the fractional advectiondispersion equation," Journal of Contaminant Hydrology, vol. 48, no. 1-2, pp. 69-88, 2001.
[44] R. Klages, G. Radons, and I. M. Sokolov, Anomalous Transport: Foundations and Applications, John Wiley \& Sons, London, 2008.

[45] K. B. Oldham and J. Spanier, The Fractional Calculus, Academic Press, New York, NY, USA, 1974, reprinted in 2006.

[46] L. C. van Rijn, "Sediment transport, part II: Suspended load transport," Journal of Hydraulic Engineering, vol. 110, no. 11, pp. 1613-1641, 1984.

[47] D. Zhong, L. Zhang, B. Wu, and Y. Wang, "Velocity profile of turbulent sediment-laden flows in open-channels," International Journal of Sediment Research, vol. 30, no. 4, pp. 285-296, 2015. 


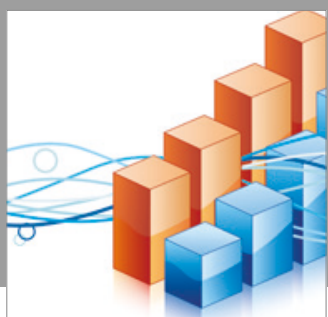

Advances in

Operations Research

vatersals

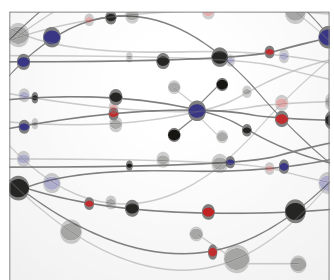

\section{The Scientific} World Journal
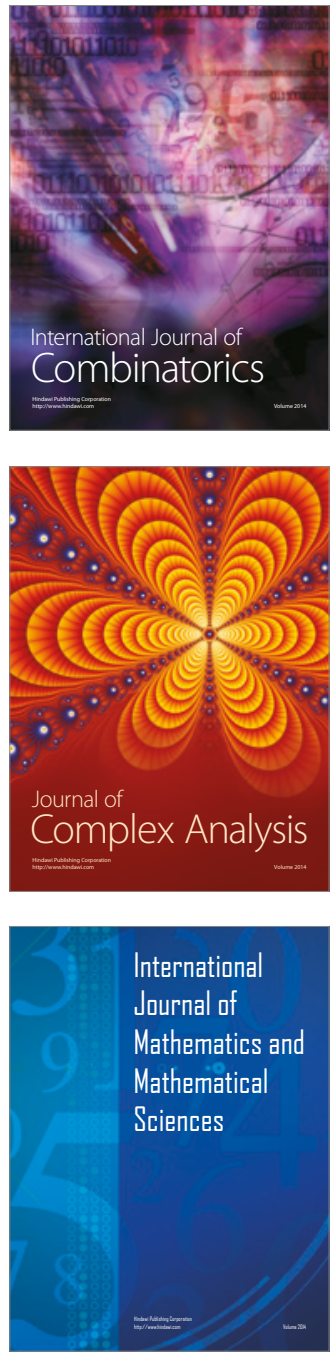
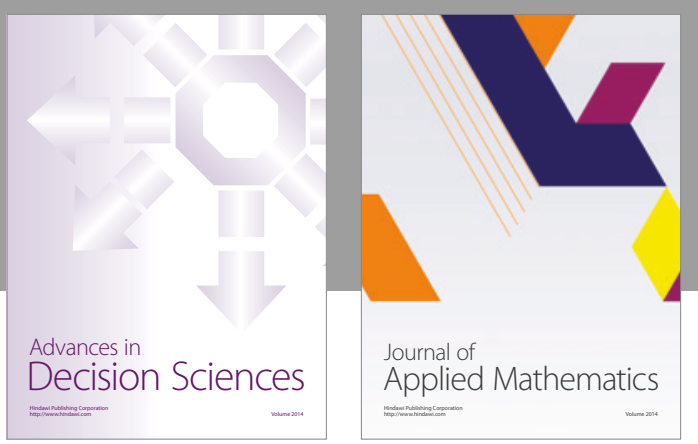

Algebra

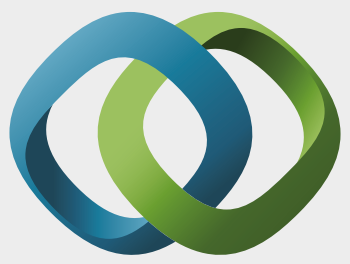

\section{Hindawi}

Submit your manuscripts at

https://www.hindawi.com
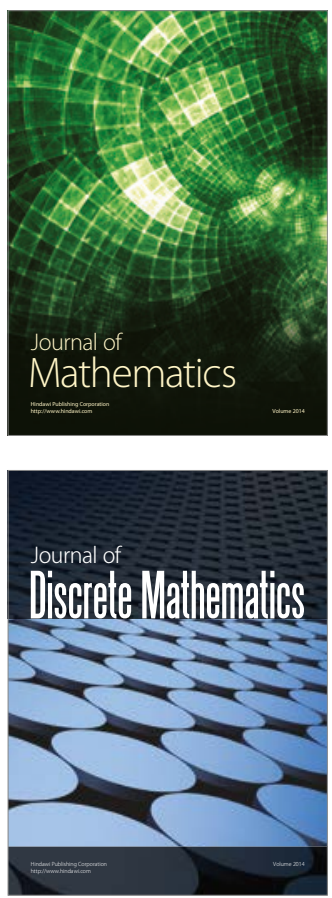

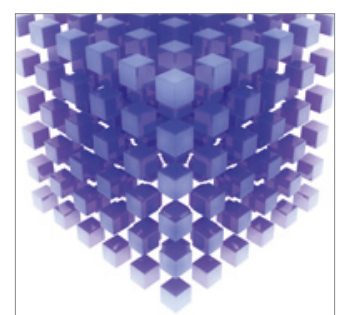

Mathematical Problems in Engineering
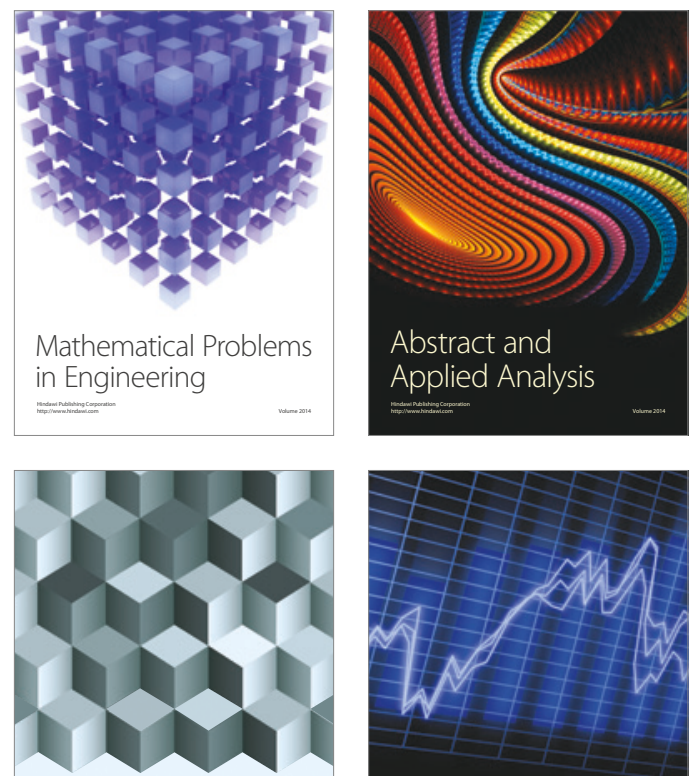

Journal of

Function Spaces

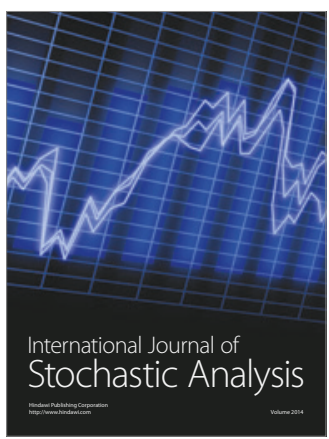

Probability and Statistics
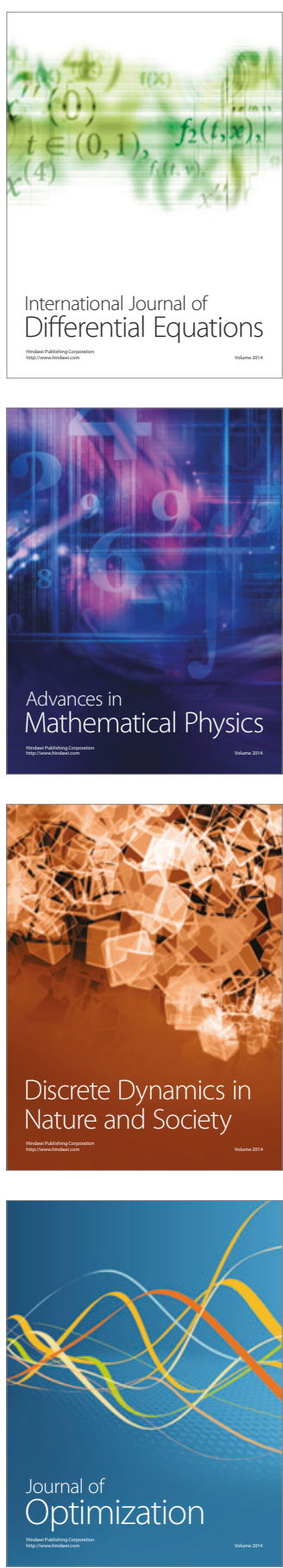